\title{
Commentary
}

\section{The time has come to target connective tissue growth factor in diabetic complications}

Dysregulation of growth factors is amongst the changes that occur in cells and tissues in diabetes. As growth factors commonly control essential biological functions, it follows that they may be critical in causing the end-organ complications of diabetes [1]. Consequently, identifying the key growth factor(s) that cause a particular type and stage of diabetic tissue damage is of potential importance in preventing and treating diabetes complications.

In this context, connective tissue growth factor (CTGF), also known as CCN2 [2], is a prime candidate. Following its identification in 1991, this $38-\mathrm{M}_{\mathrm{r}}$ cysteine-rich secreted protein has been shown to exhibit a broad spectrum of biological activities, often specific to cell and tissue type. Many of the effects of CTGF reflect cellular changes that, characteristically, occur more frequently than normal in diabetic complications. The main effects of CTGF are the regulation of extracellular matrix (ECM) accumulation [3], cell hypertrophy and mitogenesis, and in some cases, apoptosis [4], cellular adhesion and chemotaxis [5], and cell trans-differentiation [3]. The complex biological process of angiogenesis can also be promoted by CTGF [6].

CTGF gene expression and protein levels are upregulated by diabetes in tissues that are susceptible to end-organ injury. The kidney has been the main focus of CTGF research in diabetes. CTGF was found to be increased both in rodent models of diabetic renal disease, in the glomerulus $[7,8]$ and the tubulo-interstitium [9], and in human diabetes, in the same sites, involving the resident cells of the kidney [10]. Increases in CTGF renal expression correspond with the extent of glomerulosclerosis and tubulo-interstitial fibrosis,

Received: 5 January 2004 / Accepted: 21 April 2004

Published online: 28 May 2004

(C) Springer-Verlag 2004 leading to the hypothesis that CTGF contributes to early mesangial matrix expansion and the later changes of fibrosis in advanced diabetic nephropathy [10].

ECM expansion is a common feature in tissue affected by diabetes. Indeed, we have recently shown that CTGF is also increased in non-renal tissue. The increases in CTGF correspond with pathological increases in myocardial type III collagen in rodent diabetic cardiomyopathy [11]. In the diabetic apoE-deficient mouse model of atheroma, CTGF mRNA and protein levels are increased in the complex lesions at the fibrous caps, where increases in ECM are also observed [12]. In the eye of the diabetic rodent, CTGF is up-regulated in the ganglion cell layer of the retina [13]. These data suggest that CTGF may contribute to microvascular and macrovascular complications in diabetes.

The main metabolic pathways implicated in microvascular complications in diabetes cause an increase in CTGF expression. Depending on the cell type, elevated extracellular glucose, reactive oxygen species, other growth factors implicated in diabetes, e.g. TGF$\beta$ and vascular endothelial growth factor (VEGF) [14], as well as advanced glycation end-products [15] and the hexosamine pathway have all been identified as inducers of CTGF expression. In some cases, relevant response elements in the CTGF promoter that mediate particular inciting agents have been resolved. Haemodynamic factors that are increased in diabetes, such as angiotensin II [16] and static pressure, interact with the metabolic pathways in diabetes to increase renal CTGF production. Interestingly, in some models, protein kinase $\mathrm{C}$ pathways down-regulate CTGF gene expression, whilst in other systems, overexpression of protein kinase C can up-regulate CTGF [14]. Furthermore, pro-inflammatory cytokines may have the same variable effect, depending upon the diabetic model studied [14]. 
The cellular mechanism of the action of CTGF remains to be fully clarified. CTGF specifically binds integrin subtypes [2], and acts as a binding partner for a variety of growth factors $[17,18]$. In other studies, CTGF was shown to bind to the promiscuous LDL-receptor-related protein [2]. CTGF is a modular protein and its C-terminal fragments alone have been shown to confer biological activity [2], whilst its heparinbinding domain likely impacts upon its extracellular localisation, cellular targeting and activity [2]. It is unclear whether CTGF functions primarily by signalling through its own cell surface receptor, e.g. using integrins, or whether its main mechanism is the regulation of other growth factor effects. Since a receptor specific to CTGF has not yet been identified, diabetes research currently needs to target this particular growth factor.

Whilst the mechanism of CTGF action is not fully understood, the finding that CTGF interacts with other important growth factor systems provides further argument in favour of blocking CTGF in diabetes complications. Neutralisation of TGF- $\beta$ with antisense oligonucleotides or neutralising antibodies prevented structural changes of diabetic nephropathy in rodents [19]. CTGF is not only up-regulated by TGF- $\beta$, it also augments TGF- $\beta$ bioactivity [17]. Blocking VEGF attenuates proliferative and non-proliferative diabetic retinopathy [20], and in retinal cells in culture, CTGF is induced by VEGF and regulates VEGF bioactivity [18]. These data suggest that by blocking CTGF bioactivity, the TGF- $\beta$ and VEGF systems, which have already been described as critical in causing diabetic complications, will be modulated.

The arguments for the involvement of CTGF in diabetic complications are thus potent, in terms of the known bioactivities of CTGF, its up-regulation in diabetes, and its interaction with other key growth factors. Remarkably however, no in vivo studies have yet reported whether specifically blocking CTGF prevents any form of diabetes-related end-organ pathology. Studies in human primary cell cultures show that CTGF mediates, at least in part, the effect of advanced glycation end-products [21] and high glucose [22] on cell matrix accumulation. The finding that CTGF expression is inhibited in vivo in diabetic models where tissue complications are prevented, such as in nephropathy treated with the advanced glycation formation inhibitor, aminoguanidine [15], in diabetic atheroma and retinopathy treated by ACE inhibition [12, 13], and in diabetic cardiomyopathy treated with the advanced glycation cross link breaker, ALT-711 [11], provides convincing circumstantial evidence that CTGF mediates the effects of advanced glycation endproducts and angiotensin II on end-organ injury in diabetes.

Potential methods to block CTGF include the inhibition of its gene and protein expression, and the neutralisation of the effects of the active protein. Tools that are efficient in blocking CTGF in vitro include antisense oligonucleotides [23], adenoviral CTGF antisense constructs [6] to inhibit CTGF transcription, and anti-CTGF neutralising antibodies [21], which thereby block protein bioactivity. Whilst tissue and cell type targeting can be difficult to achieve, such tools used in vivo should show whether blocking CTGF is effective in preventing diabetic tissue damage. If the animal studies are successful, tools such as humanised neutralising antibodies, CTGF antisense in adeno-associated or slow viruses, siRNA, and small peptides may provide blocking methods that are more complete, and that minimise vector-related non-specific immune responses. In the future, small non-peptides may also be of value, to inhibit receptor function. Such approaches may ultimately be useful in clinical studies in humans.

A serious concern is that the growth factors to be inhibited for prevention of diabetes complications may be essential for normal biological functions. CTGF may have some advantage over other growth factors in this respect. TGF- $\beta$ has important functions in immune surveillance and suppression, and in inhibiting epithelial cell proliferation, in addition to its role in ECM regulation [24]. Indeed, TGF- $\beta$ knock-out animals die from immune system overactivity [25]. In parallel, VEGF isoforms are thought to have an important function in normal retinal development [20]. Whilst CTGF can augment the bioactivity of TGF- $\beta$ in terms of its effects on ECM, it has not been shown to suppress immune function. Furthermore, at normal tissue levels, CTGF has minimal effects on epithelial cell growth, consistent with the view that CTGF may be a more specific target for diabetic complications than TGF- $\beta$ [25]. It is also possible that blocking CTGF will attenuate the adverse effects of VEGF bioactivity in diabetic retinopathy, but will not completely block VEGF function in normal biological processes. Since CTGF gene deletion/knock-out mice die at birth from respiratory failure secondary to cartilage maldevelopment, it may be preferable for CTGF to be inhibited in a manner that is specific to tissue and time. In addition, CTGF is thought to have an important developmental role for the kidneys [4], bones and cartilage [26], and is active in wound healing [27].

As well as being a therapeutic target, CTGF may be a valuable marker for detecting early diabetic complications. Urinary CTGF excretion in people with diabetes increases in parallel with progressive renal dysfunction [28, 29]. Apparently robust CTGF assays exist, and it is hoped that a commercial assay will soon be available. Intriguingly, CTGF steady-state mRNA levels in renal biopsies of patients with Type 1 diabetes may be able to predict which individuals will develop diabetic complications over time, even in the absence of abnormal histopathology at the time of the initial biopsy [30]. Thus, measurement of CTGF in tissue samples and biological fluids may prove useful 


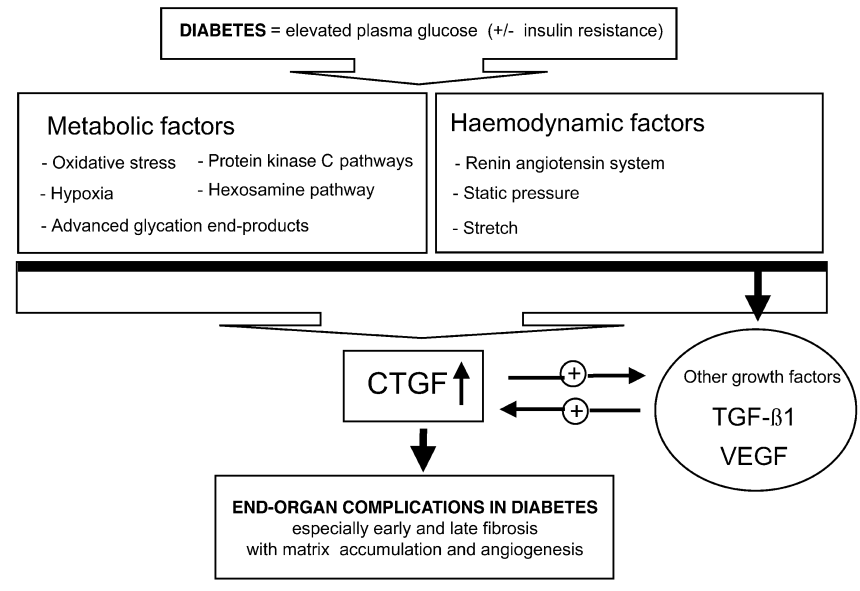

Fig. 1. A CTGF-centred view of diabetes complications

in predicting imminent and progressive diabetic complications, and in monitoring response to therapy.

This body of data collectively implicates CTGF in diabetic complications, not only in the kidney but also in non-renal tissue. However, the data are circumstantial, and in order to make progress in this field, observations of direct CTGF inhibition in animal models of diabetes are required. Such findings should resolve the issue of whether directly modulating this growth factor can safely retard, reverse or prevent diabetic complications (Fig. 1).

\section{S. M. Twigg}

Discipline of Medicine, University of Sydney,

New South Wales 2006, Australia

\section{E. Cooper}

Danielle Alberti Centre for Diabetes Complications,

Vascular Division, Wynn Domain,

Baker Heart Research Institute, Melbourne, Victoria, Australia

\section{E. Cooper}

Department of Medicine, Pathology and Immunology,

Eastern Medical School, Monash University, Victoria,

Australia

\section{References}

1. Flyvbjerg A (2000) Putative pathophysiological role of growth factors and cytokines in experimental diabetic kidney disease. Diabetologia 43:1205-1223

2. Brigstock DR (2003) The CCN family: a new stimulus package. J Endocrinol 178:169-175

3. Gore-Hyer E, Shegogue D, Markiewicz M et al. (2002) TGF-beta and CTGF have overlapping and distinct fibrogenic effects on human renal cells. Am J Physiol Renal Physiol 283:F707-F716

4. Dolan V, Hensey C, Brady HR (2003) Diabetic nephropathy: renal development gone awry? Pediatr Nephrol 18:75-84

5. Chen CC, Chen N, Lau LF (2001) The angiogenic factors Cyr61 and connective tissue growth factor induce adhesive signaling in primary human skin fibroblasts. J Biol Chem 276:10443-10452
6. Shimo T, Nakanishi T, Nishida T et al. (1999) Connective tissue growth factor induces the proliferation, migration, and tube formation of vascular endothelial cells in vitro, and angiogenesis in vivo. J Biochem 126:137-145

7. Murphy M, Godson C, Cannon S et al. (1999) Suppression subtractive hybridization identifies high glucose levels as a stimulus for expression of connective tissue growth factor and other genes in human mesangial cells. J Biol Chem 274:5830-5834

8. Riser BL, Denichilo M, Cortes P et al. (2000) Regulation of connective tissue growth factor activity in cultured rat mesangial cells and its expression in experimental diabetic glomerulosclerosis. J Am Soc Nephrol 11:25-38

9. Wang S, Denichilo M, Brubaker C, Hirschberg R (2001) Connective tissue growth factor in tubulointerstitial injury of diabetic nephropathy. Kidney Int 60:96-105

10. Ito Y, Aten J, Bende RJ et al. (1998) Expression of connective tissue growth factor in human renal fibrosis. Kidney Int 53:853-861

11. Candido R, Forbes JM, Thomas MC et al. (2003) A breaker of advanced glycation end products attenuates diabetes-induced myocardial structural changes. Circ Res 92:785-792

12. Candido R, Jandeleit-Dahm KA, Cao Z et al. (2002) Prevention of accelerated atherosclerosis by angiotensinconverting enzyme inhibition in diabetic apolipoprotein E-deficient mice. Circulation 106:246-253

13. Tikellis C, Cooper ME, Twigg SM, Burns WC, Tolcos M (2004) Connective tissue growth factor is up-regulated in the diabetic retina: amelioration by ACE inhibition. Endocrinology 145:860-866

14. Blom IE, Goldschmeding R, Leask A (2002) Gene regulation of connective tissue growth factor: new targets for antifibrotic therapy? Matrix Biol 21:473-482

15. Twigg SM, Cao Z, McLennan SV et al. (2002) Renal connective tissue growth factor induction in experimental diabetes is prevented by aminoguanidine. Endocrinology 143:4907-4915

16. Ruperez M, Lorenzo O, Blanco-Colio LM, Esteban V, Egido J, Ruiz-Ortega M (2003) Connective tissue growth factor is a mediator of angiotensin II-induced fibrosis. Circ Res 1081:499-505

17. Abreu JG, Ketpura NI, Reversade B, De Robertis EM (2002) Connective-tissue growth factor (CTGF) modulates cell signalling by BMP and TGF-beta. Nat Cell Biol 4:599-604

18. Inoki I, Shiomi T, Hashimoto G et al. (2002) Connective tissue growth factor binds vascular endothelial growth factor (VEGF) and inhibits VEGF-induced angiogenesis. FASEB J 16:219-221

19. Ziyadeh FN, Hoffman BB, Han DC et al. (2000) Longterm prevention of renal insufficiency, excess matrix gene expression, and glomerular mesangial matrix expansion by treatment with monoclonal antitransforming growth factorbeta antibody in db/db diabetic mice. Proc Natl Acad Sci USA 97:8015-8020

20. Caldwell RB, Bartoli M, Behzadian MA et al. (2003) Vascular endothelial growth factor and diabetic retinopathy: pathophysiological mechanisms and treatment perspectives. Diabetes Metab Res Rev 19:442-455

21. Twigg SM, Joly AH, Chen MM et al. (2002) Connective tissue growth factor/IGF-binding protein-related protein-2 is a mediator in the induction of fibronectin by advanced glycosylation end-products in human dermal fibroblasts. Endocrinology 143:1260-1269

22. Lam SC, Geest RN van der, Verhagen NA et al. (2003) Connective tissue growth factor and igf-I are produced by human renal fibroblasts and cooperate in the induction of collagen production by high glucose. Diabetes 52:2975-2983 
23. Abdel-Wahab N, Weston BS, Roberts T, Mason RM (2002) Connective tissue growth factor and regulation of the mesangial cell cycle: role in cellular hypertrophy. J Am Soc Nephrol 13:2437-2445

24. Gupta S, Clarkson MR, Duggan J, Brady HR (2000) Connective tissue growth factor: potential role in glomerulosclerosis and tubulointerstitial fibrosis. Kidney Int 58:1389-1399

25. Goldschmeding R, Aten J, Ito Y, Blom IE, Rabelink T, Weening JJ (2000) Connective tissue growth factor: just another factor in renal fibrosis? Nephrol Dial Transplant 15:296-299

26. Ivkovic S, Yoon BS, Popoff SN et al. (2003) Connective tissue growth factor coordinates chondrogenesis and angiogenesis during skeletal development. Development 130: 2779-2791

27. Mori R, Kondo T, Ohshima T, Ishida Y, Mukaida N (2002) Accelerated wound healing in tumor necrosis factor receptor p55-deficient mice with reduced leukocyte infiltration. FASEB J 16:963-974
28. Riser BL, Cortes P, DeNichilo M et al. (2003) Urinary CCN2 (CTGF) as a possible predictor of diabetic nephropathy: preliminary report. Kidney Int 64:451-458

29. Gilbert RE, Akdeniz A, Weitz S et al. (2003) Urinary connective tissue growth factor excretion in patients with type 1 diabetes and nephropathy. Diabetes Care 26:26322636

30. Adler SG, Kang SW, Feld S et al. (2002) Can glomerular mRNAs in human type 1 diabetes be used to predict transition from normoalbuminuria to microalbuminuria? Am J Kidney Dis 40:184-188

S. M. Twigg ( )

Discipline of Medicine, University of Sydney,

New South Wales 2006, Australia

E-mail: stwigg@med.usyd.edu.au

Tel.: +61-2-95156150

Fax: +61-2-95161273 